Ann. Biol. anim. Bioch. Biophys., 1979, 19 (1A), 73-78.

\title{
Variations de l'insulinémie chez la brebis en fin de gestation et début de lactation. Influence de l'état nutritionnel en fin de ges- tation
}

\author{
par J. GRIZARD, M. TISSIER *, M. THÉRIEZ *, R. PION \\ avec la collaboration technique de M. C. VALLUY, A. BRELURUT *, J. LEROUX * \\ Laboratoire d'Etude du Métabolisme azoté \\ * Laboratoire de la Production ovine \\ I.N.R.A., Theix, Saint-Genès Champanelle, 63110 Beaumont.
}

Summary. Plasma insulin in ewes during pregnancy and lactation. Effect of nutritional state during late pregnancy.

Twenty-eight Limousine ewes were used in a factorial experiment. The experimental treatments included two levels of energy intake during 7 weeks prepartum (high : $\mathrm{H}$; low : B) and two states of body reserves at 8 weeks prepartum (fat: $G$; lean : $M$ ). The animals bore or were suckling either twins (D) or singles (S). Preprandial and postprandial plasma insulin were recorded at 38,24 and 10 days prepartum and at 5, 13, 26 and 40 days postpartum. During pregnancy there was a high level of plasma insulin in the lean ewes bearing twins and receiving the high level of energy intake (group MHD) (fig. 1). Plasma insulin decreased during late pregnancy and increased at the beginning of lactation.

Le but du présent travail est de voir dans quelle mesure les modifications de l'utilisation de l'azote ef de l'énergie en fin de gestation ef début de lactation, sont la conséquence des variations observées de l'insuline plasmatique, par l'intermédiaire des principaux effets de cette hormone : accroissement du transport des acides aminés dans le muscle ef utilisation de ces derniers pour la protéosynthèse (Wool, 1972 ; Fulks, Li et Golberg, 1975), ralentissement de la néoglucogenèse hépatique (Parilla, Jimenez et Ayso-Parilla, 1976) et augmentation de l'utilisation de glucose par les tissus et de la lipogenèse.

Nous avons étudié l'insulinémie puisqu'on constate de nettes modifications du métabolisme de cette hormone à l'approche du dernier tiers de la gestation. En effet, la sécrétion d'insuline est accrue chez les ruminants (Hove ef Blom, 1976 ; Blom, Hove ef Nedkvitne, 1976 ; Schwalm ef Schultz, 1976) et les monogastriques (Sutter ef al., 1972 ; Bone et Taylor, 1976 ; Saudek, Finkowski et Knopp, 1975 ; Phelps et al., 1975 ; Kühl, Hornnes et Klebe, 1977) ; de même, l’intolérance au glucose et le besoin en insuline de la femme diabétique sont augmentés. 


\section{Matériel et méthodes.}

Les animaux utilisés et les méthodes expérimentales sont décrites en détail par ailleurs (Grizard et al., 1979).

L'insuline plasmatique est dosée par radioimmunologie selon la méthode du double anticorps, au moyen des trousses de dosage in vitro CEA-IRE-SORIN INSIK 1, l'insuline standard étant l'insuline humaine.

\section{Résultats.}

A 39 et 38 jours prepartum, la restriction énergétique entraîne des baisses des insulinémies préprandiale et postprandiale dans les lots MD (fig. 1) ; l'absence de réserves corporelles suffisantes à l'approche du dernier tiers de la gestation provoque un accroissement des insulinémies préprandiale ef postprandiale dans les lots HD. L'insulinémie préprandiale ne varie que peu entre 38 et 24 jours preparfum, à l'exception d'une augmentation dans le lot MHS ; en revanche, elle diminue à l'approche de la mise bas chez la plupart des animaux. L'insulinémie postprandiale diminue pendant la fin de la gestation, excepté dans les lots GHD, GHS et MHS.
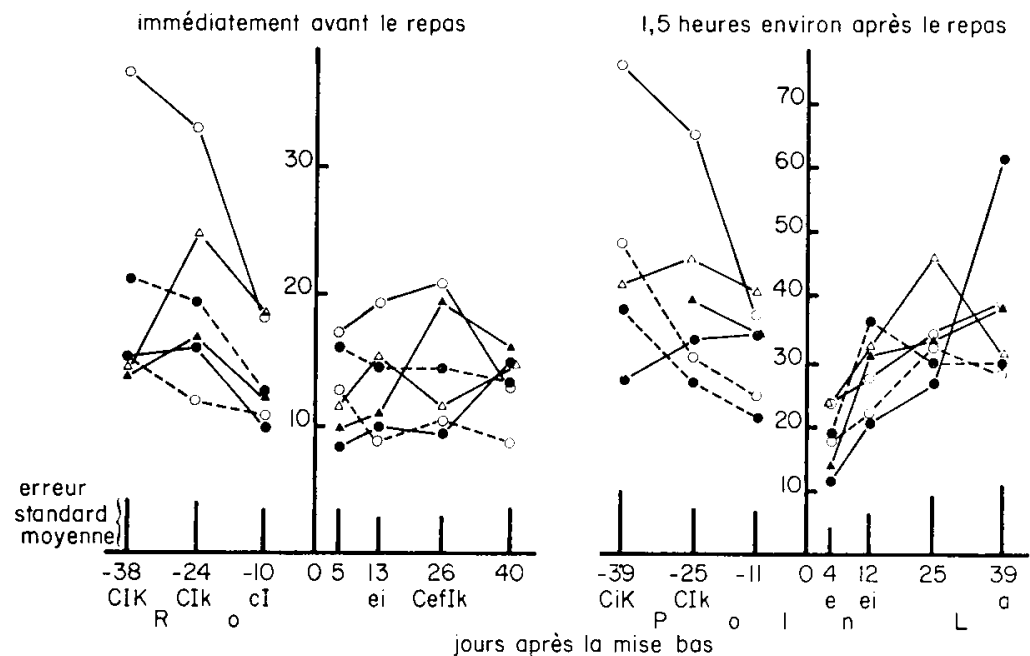

FIG. 1. - Variations de l'insulinémie $(\mu \cup$ p. $\mathrm{ml}$ ) en fin de gestation ef début de lactation.

lot GHD

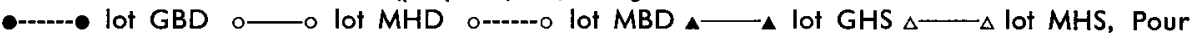
chaque temps, les lettres indiquent les différences significatives entre les teneurs constatées dans les différents lots ( $P \leqslant 0,050$ en lettres majuscules ; $0,050<P \leqslant 0,100$ en lettres minuscules); a : lot GHD différent du lot GBD ; I, i : lot MHD différent du lot MBD; C, c : lot GHD différent du lot MHD; e : lot GHS différent du lot MHS ; $f$ : lot GHD différent du lot GHS ; K, $k$ : lot MHD différent du lot MHS. Entre deux temps consécutifs, les lettres indiquent les variations significatives des teneurs ( $P \leqslant 0,050$ en lettres majuscules ; $0,050<P \leqslant 0,100$ en lettres minuscules) ; L. I ; dans le lot GHD; $n$ : dans le lot GBD ; o : dans le lot MHD ; $P$ : dans le lot $M B D: R$ : dans le lot MHS. 
A 4 et 5 jours, postpartum, ni la restriction énergétique pendant la fin de la gestation, ni l'absence de réserves corporelles suffisantes provoquent de variations notables des insulinémies préprandiale et postprandiale. Les seules variations importantes de l'insulinémie préprandiale en fonction du stade de la lactation, sont des augmentations sensibles dans les lots GHS ef MHD à 26 jours postpartum. L'insulinémie postprandiale est nettement plus faible à 4 jours postpartum que pendant la fin de la gestation. Ensuite, elle augmente sensiblement entre 4 et 25 jours postpartum ; elle ne varie plus entre 25 et 39 jours postpartum, à l'exception d'une augmentation dans le lot GHD.

Dans tous les lots, l'insulinémie postprandiale est plus forte que l'insulinémie préprandiale; celles-ci sont liées entre elles par une relation linéaire hautement significative (tabl. 1). Il y a aussi des relations linéaires significatives entre l'insulinémie ef les apports d'énergie et de matières azotées par l'alimentation.

\section{TABLEAU 1}

Comparaison de l'insulinémie postprandiale à l'insulinémie préprandiale (A) ef de l'insulinémie aux apports alimentaires (B)

\begin{tabular}{|c|c|c|c|c|c|c|}
\hline \multicolumn{7}{|c|}{ Régressions $\mathbf{Y}=\mathbf{b} \mathbf{X}+\mathbf{K}$} \\
\hline & $Y(1)$ & $\times\left({ }^{1}\right)$ & $b \pm$ écart-łype & $\mathrm{K}$ & $R^{2}$ & Signification \\
\hline A & Insulinémie postprandiale & $\begin{array}{l}\text { Insulinémie } \\
\text { préprandiale }\end{array}$ & $1,51 \pm 0,24$ & 10,5 & 0,50 & $P \leqslant 0,001$ \\
\hline B & $\begin{array}{l}\text { Insulinémie préprandiale } \\
\text { Insulinémie postprandiale } \\
\text { Insulinémie préprandiale } \\
\text { Insulinémie postprandiale }\end{array}$ & $\begin{array}{l}\text { MOD }\left({ }^{2}\right) \\
\text { MOD }\left(^{2}\right) \\
\text { PDI }\left(^{2}\right) \\
\text { PDI } \quad\left(^{2}\right)\end{array}$ & $\begin{aligned} 11,1 & \pm 5,5 \\
41,3 & \pm 11,0 \\
8,1 & \pm 4,2 \\
32,8 & \pm 8,7\end{aligned}$ & $\begin{array}{r}4,2 \\
-6,7 \\
-6,5 \\
-1,2\end{array}$ & $\begin{array}{l}0,09 \\
0,26 \\
0,08 \\
0,27\end{array}$ & $\begin{aligned} P & =0,050 \\
P & <0,001 \\
0,050 & <P<0,100 \\
P & <0,001\end{aligned}$ \\
\hline
\end{tabular}

$\mathrm{R}^{2}$ : carré du coefficient de corrélation : MOD : matière organique digestible ingérée ; PDI : protéines digestibles dans l'intestin ingérées (INRA, 1978).

(1) Moyennes observées dans chaque lot à chaque prélèvement en fin de gestation et début de lactation.

(2) Rapport entre les apports journaliers déterminés pour les deux repas précédant chaque prélèvement et les apports estimés comme nécessaires au poids des agneaux à la naissance et aux quantités de lait produites (INRA, 1978).

\section{Discussion.}

L'insulinémie après le repas est toujours plus élevée que l'insulinémie avant le repas. Ceci est dô à une augmentation de la sécrétion d'insuline (Trenkle, 1971) sous l'effet des produits de la digestion (acides gras volatils, glucose, acides aminés indispensables) (Fajans et al., 1967 ; Horino et al., 1968 ; Overfield et Trenkle, 1976) asso-iés à l'accroissement de l'activité nerveuse parasympathique (Bassett, 1975 ; Porte ef al., 1975) et de la sécrétion d'hormones intestinales insulinosécrétrices (Grossman, 1977).

\section{1. - Pendant la fin de la gestation.}

Les besoins en glucose sont très supérieurs aux quantités de glucose absorbées et sont couverts en grande partie par la néoglucogenèse ; de même, à l'approche de la 
mise bas, les besoins en matières azotées dépassenf les apports par l'alimentation (INRA, 1978 ; Grizard et al., 1979).

a) Influence de la sous-alimentation énergétique. - L'accroissement de la néoglucogenèse à partir de l'alanine, sous l'effel de la sous-alimentation énergétique, ne permet pas de compenser totalement l'augmentation du déficit en glucose (Grizard et al., 1979). Les baisses de l'insulinémie ne sont pas uniquement la cause de l'accroissement de la néoglucogenèse à partir de l'alanine puisque d'une part elles ne sont pas constatées chez tous les animaux ef d'autre part d'autres hormones que l'insuline peuvent stimuler la néoglucogenèse (glucagon, glucocorticoïdes, catécholamines) (Exton et al., 1970 ; Brockman et al., 1975). Elles sont dues en partie à une baisse de la sécrétion d'insuline sous l'effet des réductions de la glycémie, des teneurs sanguines en certains acides aminés libres indispensables insulinosécréteurs (leucine, valine, phénylalanine, tyrosine et thréonine) (Grizard ef al., 1979), de l'absorption d'acides gras volatils insulinosécréteurs (propionate, butyrate) et de l'activité nerveuse parasympathique sous l'effet de la diminution de la durée des repas. Elles confirment les constatations de Prior ef Christenson (1976) et sont à rapprocher des réductions de l'insulinémie à la suite de la diminution de l'apport alimentaire chez le mouton (Bassett, 1974) ou de la part d'aliment concentré dans la ration chez le mouton (Lofgren et Warner, 1972) et la vache au début de la lactation (Walker et Elliot, 1973 ; Jenny et Polan, 1975 ; Evans, Buchanan-Smith et MacLeod, 1975).

b) Influence de l'absence de réserves corporelles suffisantes. - L'absence de réserves corporelles suffisantes au début du dernier tiers de la gestation est associée à une réduction du déficit en glucose (Grizard ef al., 1979). Les augmentations significatives de l'insulinémie pour les lots HD peuvent résulter d'une augmentation de la sécrétion d'insuline sous l'effet des augmentations de la glycémie et des teneurs sanguines de certains acides aminés libres indispensables insulinosécréteurs (leucine, méthionine) (Grizard et al., 1979).

c) Influence du stade de gestation. - A l'approche de la mise bas, la captation de glucose et de matières azotées par l'utérus augmente (INRA, 1978) ; en revanche, l'utilisation du glucose ainsi que le catabolisme des acides aminés ef leur incorporation dans les protéines sont sans doute diminués dans les tissus maternels (Grizard ef al., 1979). Les valeurs élevées de l'insulinémie aux deux tiers environ de la gestation résultent aussi bien d'un accroissement de la synthèse que de la sécrétion d'insuline (Bone et Taylor, 1976). Elles ont d'ailleurs été observées chez la brebis (Hove et Blom, 1976 ; Blom, Hove et Nedkvitne, 1976), la vache (Hove, 1974 ; Schwalm et Schultz, 1976) et la ratte (Sutter et al., 1972 ; Saudek, Finkowski et Knopp, 1975). Les réductions de l'insulinémie à l'approche de la mise bas (Blom, Hove et Nedkvitne, 1976 ; Schwalm et Schultz, 1976) peuvent être dues à l'effet d'une réduction de l'absorption d'acides gras volatils insulinosécréteurs, provoquée par une baisse des fermentations dans le rumen, associée à l'augmentation du volume de l'utérus. Elles peuvent expliquer la baisse de l'utilisation de glucose et de la protéosynthèse dans les tissus maternels puisque l'insuline accroît l'utilisation de glucose par les cellules et accroît la synthèse et réduit le catabolisme protéique (Wool, 1972 ; Fulks, Li et Golberg, 1975). 
2. - Pendant le début de la lactation.

Pendant les deux premières semaines de la lactation, le déficit en glucose est moins intense que pendant la fin de la gestation et les besoins en la plupart des acides aminés indispensables et semi-indispensables sont supérieurs aux apports par l'alimentation (INRA, 1978 ; Grizard et al., 1979). Les faibles valeurs de l'insulinémie postprandiale un peu après la mise bas favorisent sans doute l'élévation de la mobilisation des protéines et lipides corporels puisque l'insuline stimule la protéosynthèse et la lipogenèse. Les fortes augmentations de l'insulinémie postprandiale pendant les 4 premières semaines environ de la lactation peuvent être dues à un accroissement de la sécrétion d'insuline sous l'action d'une élévation de l'absorption d'acides gras volatils insulinosécréteurs associée à l'augmentation de l'activité fermentaire dans le rumen. Elles confirment les constatations de Koprowski et Tucker (1973) et Smith, Hansel et Coppock (1976) effectuées chez la vache pendant le premier mois de la lactation.

\section{Conclusion.}

Les réductions de l'insulinémie, provoquées par une sous-alimentation énergétique en fin de gestation, ne sont pas la seule cause de l'accroissement de la néoglucogenèse à partir de l'alanine. En revanche, les baisses de l'insulinémie à l'approche de la mise bas peuvent expliquer une baisse de l'utilisation du glucose et de la protéosynthèse dans les tissus maternels. De même, les faibles valeurs de l'insulinémie pendant la première semaine de la lactation, provoquent sans doute la forte mobilisation des protéines ex lipides corporels.

Reçu en mars 1978.

Accepté en septembre 1978.

\section{Références}

BASSETT J. M., 1974. Diurnal patterns of plasma insulin, growth hormone, corticosteroid and metabolite concentrations in fed and fasted sheep. Aust. J. biol. Sci., 27, 167-181.

BASSETT J. M., 1975. Dietary and gastrointestinal control of hormones regulating carbohydrate metabolism in ruminants, 383-398. In Mc DONALD I. W., WARNER A. C. I., Digestion and metabolism in the ruminant. Ed Univ. New England.

BLOM A. K., HOVE K., NEDKVITNE J. J., 1976. Plasma insulin and growth hormone concentrations in pregnant sheep. II - Post absorptive levels in mid and late pregnancy. Acta endocr., 82, 553-560.

BONE A. J., TAYLOR K. W., 1976. Metabolic adaptation to pregnancy shown by increased biosynthesis of insulin in islets of Langerhans isolated from pregnant rats. Nature, 262, 501-502.

BROCKMAN R. P., BERGMAN E. N., JOO P. K., MANNS J. G., 1975. Effects of glucagon and insulin on net hepatic metabolism of glucose precursors in sheep. Am. J. Physiol., 229, 1344-1350.

EVANS E., BUCHANAN-SMITH J. G., MacLEOD G. K., 1975. Postprandial patterns of plasma glucose, insulin and volatile fatty acids in ruminants fed low and high roughage diets. $J$. anim. Sci., 41, 1474-1479.

EXTON J. H., MALleTte L. E., JefFERSON L. S., WONG E. H. A., friedMANN N.,. MILLER T. B., PARK C. R., 1970. The hormonal control of hepatic gluconeogenesis. Rec. Progr. Horm. Res., 26, 411-461. 
FAJANS S. S., FLOYD J. C., KNOPF R. F., CONN J. W., 1967. Effect of amino acids and proteins on insulin secretion in man. Rec. Progr. Horm. Res., 23, 617-662.

FULKS R. M., LI J. B., GOLBERG A. L., 1975. Effect of insulin, glucose and amino acids on protein turnover in rat diaphragm. J. biol. Chem., 250, 290-298.

GRIZARD J., TISSIER M., CHAMPREDON C., PRUGNAUD J., PION R., 1979. Variations des teneurs sanguines en acides aminés libres, urée et glucose chez la brebis en fin de gestation et début de lactation. Influence de l'état nutritionnel en fin de gestation. Ann. Biol, anim. Bioch. Biophys., 19, 55-71.

GROSSMAN M. I., 1977. Physiological effects of gastrointestinal hormones. Fed. Proc., 36, 19301932.

HORINO M., MACHLIN L. J., HERTELENDY F., KIPNIS D. M., 1968. Effect of short-chain fafty acids on plasma insulin in ruminant and non ruminant species. Endocrinology, 83, 118-128.

HOVE K., 1974. Nocturnal plasma insulin levels in cows with varying levels of plasma ketone bodies; relations to plasma sugar and acetoacetate. Acta endocr., 76, 513-524.

HOVE K., BLOM A. K., 1976. Plasma insulin and growth hormone concentrations in pregnant sheep. I. - Diurnal variations in mid and late pregnancy. Acta endocr., 82, 544-552.

I.N.R.A., 1978. Alimentation des ruminants, Ed. INRA Publications (route de Saint Cyr), 78000 Versailles.

JENNY B. F., POLAN C. E., 1975. Postprandial blood glucose and insulin in cows fed high grain. J. Dairy Sci., 58, 512-514.

KOPROWSKI J. A., TUCKER H. A., 1973. Bovine serum growth hormone, corticoids and insulin during lactation. Endocrinology, 93, 645-651.

KUHL C., HORNNES P., KLEBE J. G., 1977. Effect of pregnancy on the glucagon response to profein ingestion. Horm. Metab. Res., 9, 206-209.

LOFGREN P. A., WARNER R. G., 1972. Relationship of dietary caloric density on certain blood metabolites to voluntary feed intake in mature wethers. J. anim. Sci., 35, 1239-1247.

OVERFIELD J. R., TRENKLE A., 1976. Nutritional control of plasma insulin levels in sheep. J. anim. Sci., 42, 1365 (abstr.).

PARILLA R., JIMENEZ M. I., AYSO-PARILLA M. S., 1976. Cellular redistribution of metabolites during glucagon and insulin control of gluconeogenesis in the isolated perfused rat liver. Arch. Bioch. Biophys., 174, 1-12.

PHELPS R. L., BERGENSTAL R., FREINKEL N., RUBENSTEIN A. H., METTZGER B. E., MAKO M., 1975. Carbohydrate metabolism in pregnancy. XIII - Relationships between plasma insulin and proinsulin during late pregnancy in normal and diabetic subjects. J. clin. Endocr. Metab., 41, 1085-1091.

PORTE D., WOODS S. C., CHEN M., SMITH P. H., ENSINCK J. W., 1975. Central factors in the control of insulin and glucagon secretion. Pharm. Bioch. Behov., 3, suppl. 1, 127-133.

PRIOR R. L., CHRISTENSON R. K., 1976. Influence of dietary energy during gestation on lambing performance and glucose metabolism in Finn-cross ewes. J. anim. Sci., 43, 1114-1124.

SAUDEK C. D., FINKOWSKI M., KNOPP R. H., 1975. Plasma glucagon and insulin in rat pregnancy. Role in glucose homeostasis. J. clin. Invest., 55, 180-187.

SCHWALM J. W., SCHULTZ L. H., 1976. Relationship of insulin concentration to blood metabolites in the dairy cow. J. Dairy Sci., 59, 255-261.

SMITH R. D., HANSEL W., COPPOCK C. E., 1976. Plasma growth hormone and insulin during early lactation in cows fed silage based diets. J. Dairy Sci., 59, 248-254.

SUTTER M. T., FELIX J. M., JACQUOT R., SUTTER B. C. J., 1972. Insuline immunoréactive, activité insulinique et glucose sériques de la ratte gestante. C. R. Acad. Sci. Paris Sér. D, 274, 1538-1541.

TRENKLE A., 1971. Postprandial changes in insulin secretion rate in sheep. J. Nutr., 101, 1099-1103.

WALKER C. K., ELLIOT J. M., 1973. Effect of roughage restriction on serum insulin in the dairy cow. J. Dairy Sci., 56, 375-377.

WOOL I. G., 1972. Insulin and the regulation of protein synthesis in muscle. Proc. Nufr. Soc., 31, 185-191. 